\title{
Grasp-stability analysis of a two-phalanx isotropic underactuated finger
}

\author{
R. Rizk, S. Krut, E. Dombre \\ LIRMM, Univ. Montpellier 2, CNRS, 161 rue Ada, 34392 Montpellier, France \\ rizk/krut/dombre@lirmm.fr
}

\begin{abstract}
Many underactuated grippers with fingers have been developed these last years. Their drawback is that they only ensure conditionally grasp stability. This paper presents a study of the grasp stability of an isotropic underactuated finger, which is made by two phalanxes and uses cams and tendon for actuation. The paper presents also a study of the internal forces developed in the transmission chains. The proposed model serves for the gripper using as for part dimensioning.
\end{abstract}

Keywords: force isotropy, grasp stability, underactuation.

\section{INTRODUCTION}

$\mathrm{M}$ OST industrial applications require grasping. A good gripper must be able to adapt itself on the grasped object whatever its shape. A better shape adaptation increases the number of contact points between the gripper and the object. Increasing the number of contact points provides a better repartition of contact forces, which ensures a better stability of grasp and prevents from deterioration of the grasped object. A gripper which provides a uniform contact pressure is said to be isotropic [1].

The best gripper is the human hand. Reaching the human finger dexterity and adaptation capabilities requires the control of a lot of actuators and sensors [2]. Advanced robotic hands have been developed with this requirement in mind. Many dexterous hands having several actuators (more than six) can be mentioned: the Utah/MIT hand [3], the Stanford/JPL Salisbury's hand [4], the Belgrad hand revisited at USC [5], the DLR hand [6].

The dexterity can also be obtained by underactuation, which consists in equipping the finger with fewer actuators than the number of degrees of freedom (DOF)[7]. Thus, the shape of the grasped object and the static equilibrium govern the gripper configuration. In [8], the advantages of such an underactuated gripper over a simple parallel one are presented. In [9], an underactuated hand with three fingers is presented. Each finger has two phalanxes and one actuator. A special mechanism is added in order to allow the distal phalanxes to be maintained orthogonal to the palm when precision grasps are performed. An artificial hand mimicking the human hand is presented in [10]. This hand

Manuscript submitted 27 March, 2007. has partially underactuated fingers. Each finger has three phalanxes. A coupling is introduced between the motion of the middle and distal phalanxes. The drawback of underactuation is that it guarantees conditional stability only. A non stable grasp led to ejection of the grasped object. Moreover, it is difficult to control the contact pressure. Most underactuated grippers reported in the literature $[1,2,7-22]$ display isotropy only in certain configurations. In this paper, we present a study of the grasp stability of a two-phalanx isotropic gripper. In section 2, we review several works that have been done on underactuated fingers and we define force isotropy. In section 3, we present the design and characteristics of a finger that we have designed. A kinetostatic analysis of this finger is realized in Section 4. The analysis of contact forces and internal forces allows us to determine the grasp stability of the finger and the efforts exerted on the passive elements respectively. The paper ends by a conclusion and propositions of further work.

\section{UNDERACTUATION IN ROBOTIC FINGERS AND FORCE ISOTROPY}

Underactuation consists in reducing the number of actuators with respct to the number of DOF[7]. The objective is to adapt the gripper on the grasped object whatever its shape. Underactuation can be realized by using differential, compliant or triggered mechanism. In order to avoid the deterioration of the grasped object, contact pressure must be as homogenous as possible. A gripper which ensures a uniform pressure is said to be isotropic [1]

\section{A. Underactuation in robotic fingers}

The concept of underactuation in robotic hands should not be confused with underactuation in robotic system. The joint coordinates of an underactuated robot are indirectly controllable. The cart and pole system (inverted pendulum) [24] is underactuated. The pendulum has four DOF among which two are actuated and two are governed by the system dynamics. In an underactuated finger, joint angles are imposed by the grasped object shape, the static equilibrium and passive components (spring, mechanical limits,...). The main difference between both concepts is that in robotic systems DOF are governed by the dynamics and in robotic fingers by the statics. However, if in robotic systems the 
number of DOF is the rank of the Jacobian matrix as in the Grübler formula [25], in underactuated fingers the number of DOF represents the number of parameters that define the finger configuration. These parameters are also called "configuration variables" [15]. The BarrettHand [16] can also be considered as underactuated since the folding angle of each finger depends not only on the actuator but also on the shape of the grasped object, thus there is one actuator and two DOF. In addition to the classical parameters known in robotics, the notion of kinematic irreversibility and the use of flexible bodies must be introduced. The gripper developed for the Canadian Space Agency is said to have 10 DOF [13], but the backdrivability of each finger has been removed thanks to worm gears. The underactuated prosthetic hand of Arts Lab (Italy) [14] relies on an "adaptive grasp mechanism" designed to share the forces throughout each finger using compression springs.

Underactuation can be achieved by using differential, compliant or triggered mechanisms. Differential mechanisms can be based on linkage system $[2,7,8,10,13$, 22] or on tendon-actuated mechanisms [1, 9, 11, 12, 15, 17, $20,21,22]$. Tendon systems are limited to small grasp forces. They induce friction and elasticity. Linkage mechanisms are more efficient for applications with large grasp forces but are relatively more bulky.

In triggered mechanisms, once the torque exceeds a certain value, the joint locks. On the Barrett Hand, the transmission is disengaged and an irreversible mechanism prevents backdrivability of the joint [18]. In Lee's hand [19], this is achieved by the use of automatic brakes.

It is also possible to reduce the number of actuators by introducing compliance for each DOF. In [14], each finger is linked to a common actuator through compliant springs. If one of the fingers is blocked, the other ones are not blocked for a certain range. The stiffness of the springs must be sufficiently small in order to allow adaptation. Therefore, the stiffness of the grasp is limited.

Differential mechanisms allow control of the contact forces on the phalanxes in contact, but require high actuator torques and high internal loads in the gripper structure, as they guarantee conditional grasp stability only. Compliant mechanisms are capable of adapting themselves to the shape of the grasped object and are always in equilibrium, but if contact forces depend on spring stiffness then they are noncontrollable. Triggered mechanisms provide always a stable grasp on a fixed object since there is no sliding, but they are not able to follow a moving object once the contact with this object is lost since the motion of the proximal phalanx is blocked.

Robotic or prosthetic fingers in which the motion of all phalanxes is mechanically coupled [23, 29-30] are not underactuated. They have one actuator and one DOF. The motion is determined by the design and there is no shape adaptation.

\section{B. Force isotropy}

High differences between contact forces induce bad stress-distribution on the grasped object, meaning bad distribution of deformation, and consequently stressconcentration and deterioration. It is known that a hydrostatic pressure induces a Von-Mises stress null [31]. Hence a body subjected to hydrostatic pressure does not present any risk of deterioration. A gripper which ensures uniform contact pressure is said to be "isotropic" [1]. In [20] a gripper which ensures the same contact force on the middle of each phalanx is presented. Since both phalanxes have the same length, it is possible to consider the gripper as isotropic. This is true since the contact force is the resultant of the uniform pressure exerted on the phalanx. In this paper, we present a kinetostatic study of this finger. We analyse the contact forces, consequently the grasp stability. In a second step we study internal forces developed in the tendon and against the mechanical joint limits.

\section{FINGER DESIGN}

In a pulley-tendon finger, the torque transmission ratios are equal to the pulley radius ratios[2]. The idea is to replace the pulleys by cams (Fig.1) [20] in order to give a variable transmission ratio depending on the folding angle, hence ensuring force isotropy.

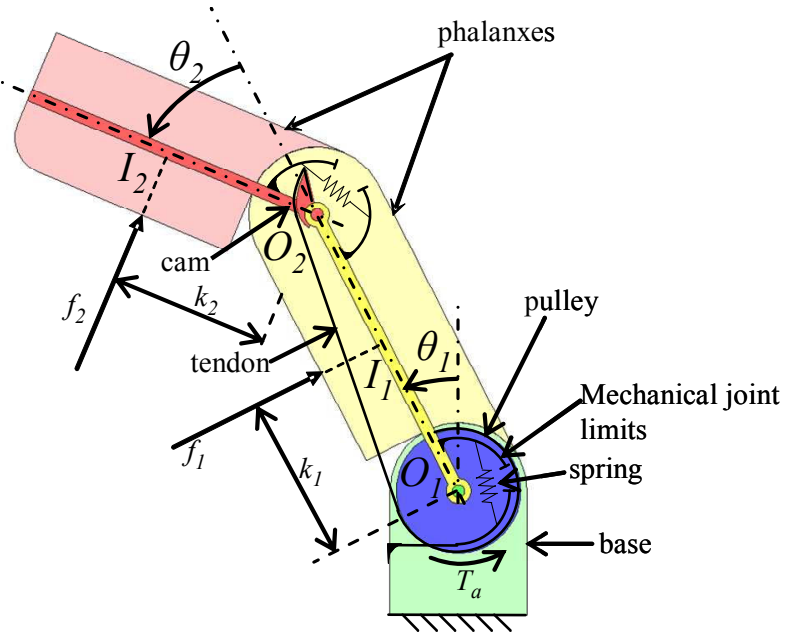

Fig. 1 - Principle of the force-isotropic underactuated finger [20]

\section{A. Transmission ratio}

Contact forces depend mainly on the actuator torque and the torque transmission ratio between unactuated joints. The equation obtained by equating both contact forces yields the formula of the required transmission ratio. The virtual power theorem gives contact forces [12]:

$$
f=\left[\begin{array}{l}
f_{1} \\
f_{2}
\end{array}\right]=\left[\begin{array}{c}
\frac{k_{2}(1+R)+R l_{1} \cos \theta_{2}}{k_{1} k_{2}} T_{a}-\frac{k_{2}+l_{1} \cos \theta_{2}}{k_{1} k_{2}} T_{2} \\
-\frac{R}{k_{2}} T_{a}+\frac{1}{k_{2}} T_{2}
\end{array}\right]
$$


where $T_{a}$ is the actuator torque, $T_{2}$ is the torque induced by the spring (neglected in practice), $k_{i} i \in\{1,2\}$ define the contact locations, $l_{1}$ is the length of phalanx $1, \theta_{2}$ is the finger folding angle, and $R$ is the transmission torque ratio between the actuator and the distal phalanx given by [20]:

$R=\frac{T_{2}}{T_{a}}=-\frac{r_{2}}{r_{1}}$

where $r_{1}$ and $r_{2}$ are the lever arms of the force in the tendon with respect to passive joint axes $O_{1}$ and $O_{2}$ respectively. Both phalanxes having the same length, force isotropy is get if and only if $f_{1}=f_{2}$, meaning that:

$\frac{k_{2}(1+R)+R l_{1} \cos \theta_{2}}{k_{1} k_{2}}=-\frac{R}{k_{1}}$

For a contact occurring at the middle of each phalanx:

$k_{1}=k_{2}=\frac{l}{2}$,

Combining equations (2) and (4) yields:

$R=\frac{-1}{4 \cos ^{2} \frac{\theta_{2}}{2}}$.

The required transmission ratio depends on $\theta_{2}$ only. The tendon transmits the force between the driving pulley and the receiving pulley. The torques ratio is that of lever arms. With circular pulleys, the ratio of lever arms is the ratio of pulleys radii. The using of pulleys with variable radii, such as cams, allows us to have a variable transmission ratio. The suitable cams are those ensure the required transmission ratio whatever the finger configuration.

\section{B. Cam profile}

Pulleys are replaced by cams providing a variable transmission torque ratio. For each $\theta_{2}$ the ratio of distances $\left(r_{1}\right.$ and $\left.r_{2}\right)$ between the tendon and $O_{1}$ and $O_{2}$ must be equal to $R$. When $\theta_{2}$ varies of a value $\Delta \theta_{2}$, the relative motion of the proximal phalanx in the frame $\left(O_{2}, x_{2}, y_{2}\right)$ of the distal phalanx is a rotation $-\Delta \theta_{2}$. Geometrically the tendon is a line. Hence, during the work of the mechanism, the tendon is carried by a set of lines. These lines turn around $\mathrm{O}_{2}$ (in $\mathrm{O}_{2} x_{2}$, $y_{2}$ ) while keeping the ratio between $r_{1}$ and $r_{2}$ equal to $R$. The cam profile is then the envelope curve of this set of lines (fig.2).

When the finger is moving, the rotations of both cams must be synchronized by the tendon. Practically, the length of the tendon unrolled on a cam must be equal to the length of the tendon rolled up on the other one. Mathematically, the synchronization corresponds to:

$d s_{1}=d s_{2}$.

where $s_{1}$ and $s_{2}$ are the arc lengths of the cams, and $d s_{i}$ denotes the differential of $s_{i}$.

Moreover, to obtain the isotropy, equation (2) must be satisfied whatever the finger configuration. Equation (3) imposes the ratio between $r_{1}$ and $r_{2}$, but does not define them. Either $r_{1}$ or $r_{2}$ must be chosen, and according to the choice the other parameter is defined. One of the pulleys can have a constant radius. This choice has the advantage to satisfying equation (6) without worrying about the relative alignement of both cams.

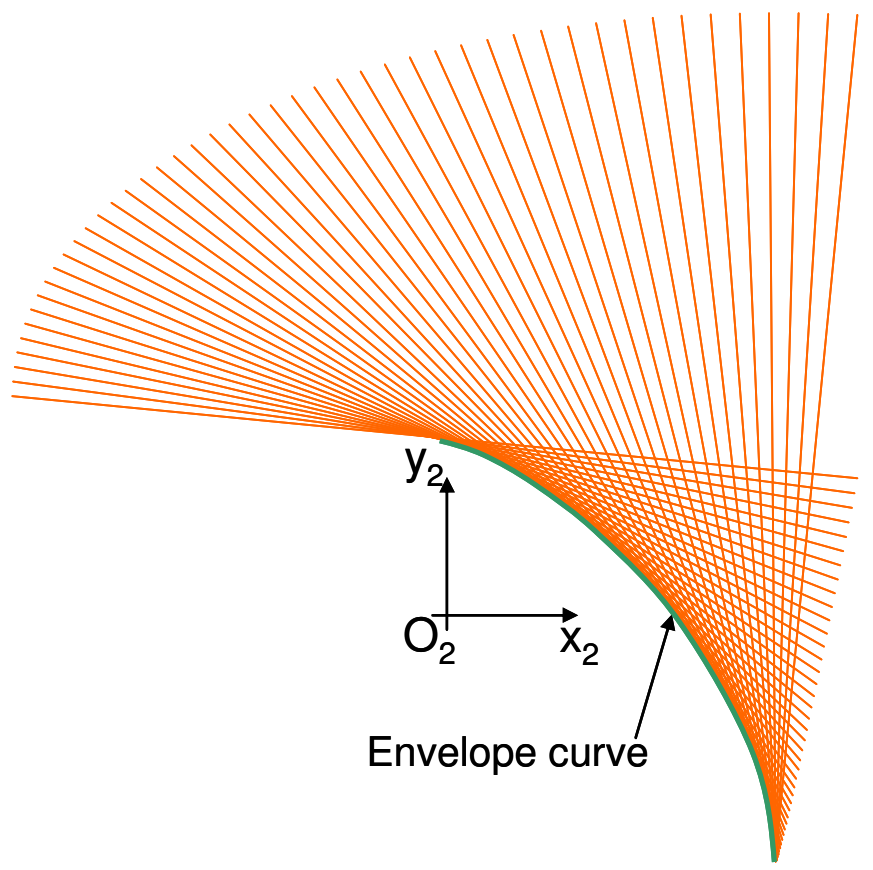

Fig 2 Set of supports of the tendon around $\mathrm{O}_{2}$

The computation of the profile gives for $l=100 \mathrm{~mm}$ and $r=20 \mathrm{~mm}$ the profile shown on Fig. 3

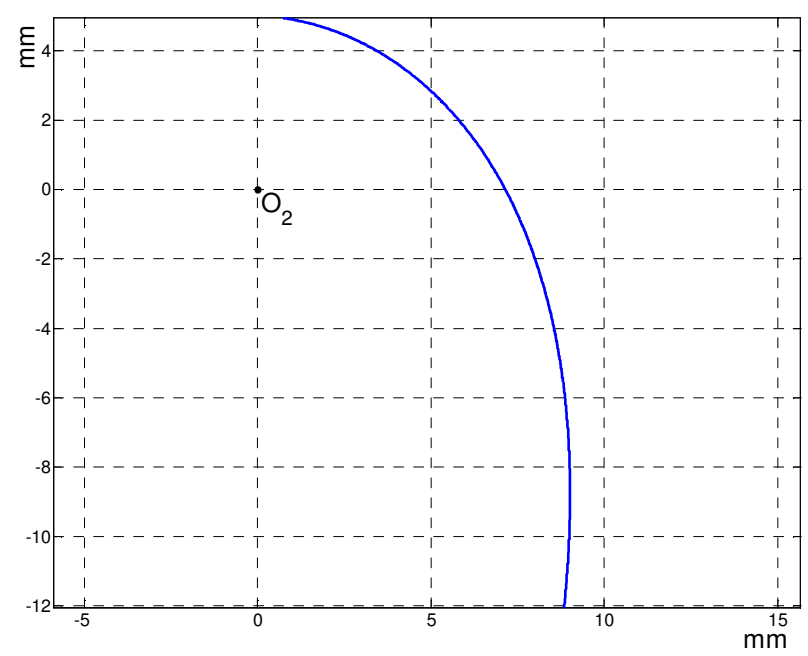

Fig. 3 Cam profile

The cam profile is convex. The tendon can wind around this profile. Let now study the grasp stability of the finger and the internal loading in the passive elements.

\section{FORCES ANALYSIS}

\section{A. Contact forces calculation}

A grasp is stable if and only if all contact forces are 
positive or null [2] (a negative force would mean that the grasped object attracts the phalanx). Two cases can be considered:

- $f_{1} f_{2} \neq 0$

- $f_{1}=0$

The first case corresponds to a grasp with both phalanxes. The gripper sizes the object with its both phalanxes. Sliding occurs until that the torque ratio is equal to $R$. Both contact forces may be different. The second case corresponds to the lost of contact with the first phalanx. The equilibrium is ensured when the torque of $f_{2}$ with respect to $O_{2}$ and $O_{1}$ have a ratio equal to $-R$.

1) Case $f_{1} f_{2} \neq 0$, contact with both phalanxes.

$f_{2}$ is always positive (equation (1)). Physically, a grasp cannot be stable without a contact with the distal phalanx. $f_{I}$ is positive if and only if:

$$
\begin{aligned}
& k_{2}(1+R)+R l \cos \theta_{2}>0 \\
& \Leftrightarrow \frac{k_{2}}{l}>-\frac{R \cos \theta_{2}}{1+R}
\end{aligned}
$$

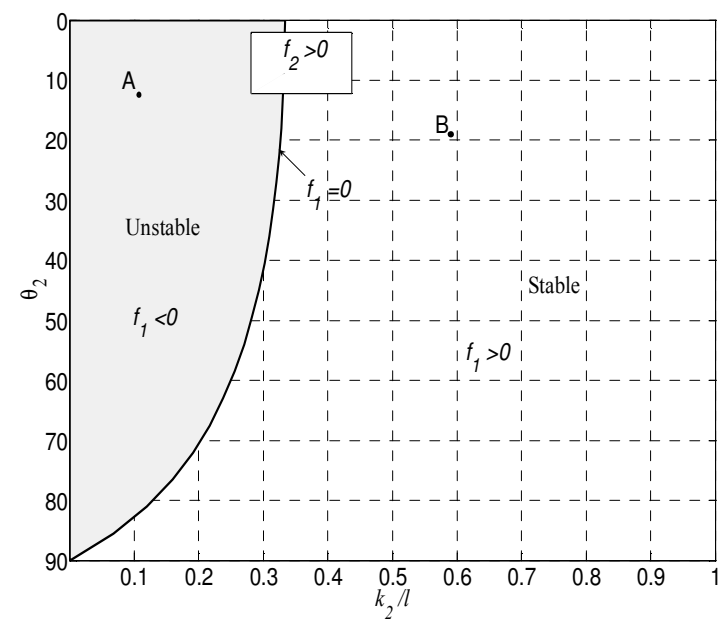

Fig. 4: Positive zone for $f_{l}$

Equation (8) defines an area in the plane $\left(k_{2} / l, \theta_{2}\right)$

(Fig.4). The grasp can be characterized by the location of the contact point on the second phalanx and the gripper folding angle. These two parameters locate a point in $\left(k_{2} / l, \theta_{2}\right)$. If the point is in the unstable zone (point A), the first phalanx loses the contact. If the contact is in stable zone (point B), the grasp is stable.

2) Case $f_{1}=0$, contact with the distal phalanx only.

This case corresponds to a contact with the second phalanx only. In this case, an unstable grasp causes sliding. The sliding can lead to a stable grasp, a fully open finger blocked by its mechanical joint limit, or an ejection.

\section{B. Diagram of stability}

The notion of diagram of stability was introduced by
Birglen [22] in order to analyse how the gripper can ensure a stable grasp. When the contact is only with the second phalanx, the grasp is stable if and only if equation (1) gives $f_{l}=0$. At the contact, the phalanx slides on the object until the condition $f_{l}=0$ is satisfied.
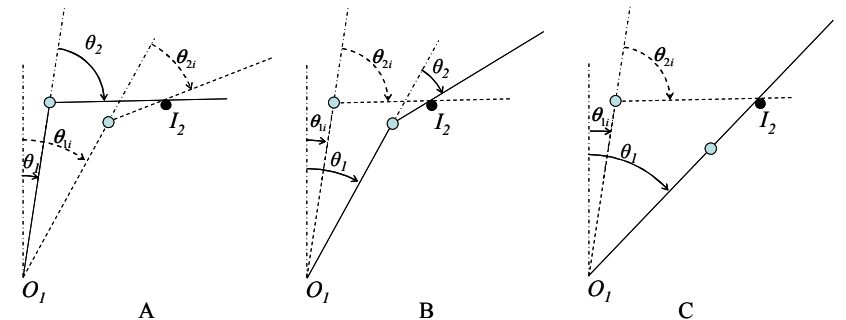

Fig. 5: Possibilties of the distal phlanx sliding on the contact point

During the sliding, the distance between $\mathrm{O}_{1}$ and the contact point $I_{2}$ (Fig.5) is constant whatever the gripper configuration. The generalized Pythagoras theorem applied on the triangle $O_{1} O_{2} I_{2}$ gives:

$\left\|\overrightarrow{\mathrm{OI}_{2}}\right\|^{2}=l^{2}+k_{2}^{2}+2 l k_{2} \cos \theta_{2}$.

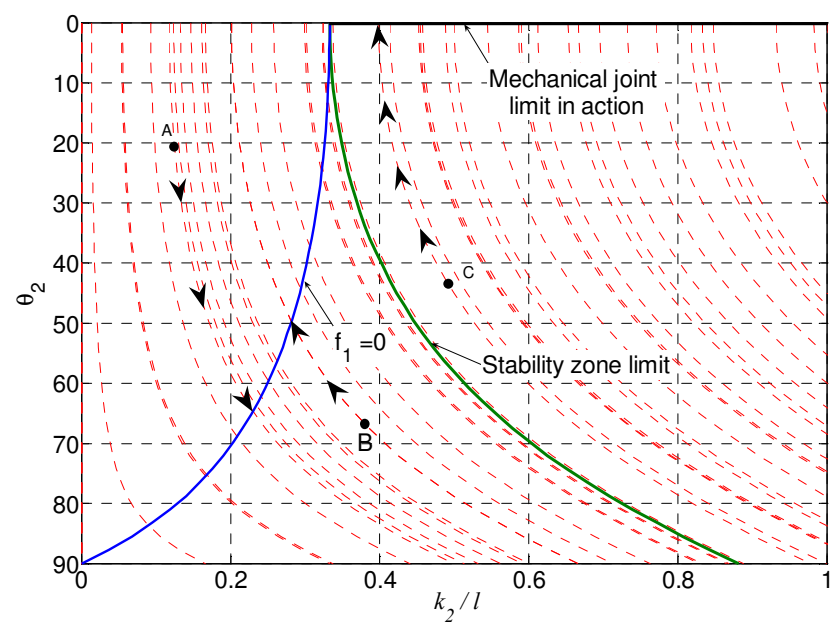

Fig. 6: Final stability of the grasp with one phalanx contact

Equation (9) defines a correspondence between $k_{2}$ and $\theta_{2}$. This correspondence may be represented by a family of curves in the plane $\left(k_{2} / l, \theta_{2}\right)$. These curves can be considered as the sliding trajectories of the gripper on the object [22].

If the contact begin with $\left(k_{2 i} / l, \theta_{2 i}\right)$ defineing a point corresponding to $f_{1}<0$ (fig. 5 case A, fig. 6 point A), then $\theta_{1}$ decreases. Hence both $\theta_{2}$ and $k_{2}$ increase. On the plane $\left(k_{2} / l\right.$, $\theta_{2}$ ) the trajectory goes toward the curve defined by $f_{l}=0$. The procedure continues until the intersection between the sliding trajectory and the curve $f_{l}=0$ where the grasp is stable.

If the contact begins when $\left(k_{2 i} / l, \theta_{2 i}\right)$ defines a point corresponding to $f_{1}>0$, then $\theta_{1}$ increases. Hence both $k_{2}$ and $\theta_{2}$ decrease. Two cases are possible:

- The sliding trajectory may intersect the curve $f_{l}=0$ before the finger is fully open (Fig. 5 case B, Fig.6 point B). The grasp is stable. 
- The sliding continues until the finger is fully open (Fig. 5 case C, Fig.6 point C). The mechanical joint limit blocks the finger. Without the mechanical joint limit, the finger would be hyper-deflected and sliding would continue until ejection.

In conclusion, with the finger proposed, there is no ejection. The grasp is stable or the mechanical joint limit prevents the ejection.

\section{Forces generated by the mechanical joint limit}

When the finger is fully open, ejection is eliminated thanks to the mechanical joint limit. The finger is in equilibrium if $T_{2}$ matches the required force to equilibrating that torque induced by $f_{2}$ at $O_{2}$. $f_{2}$ is caused by $\mathrm{T}_{\mathrm{a}}$, hence if $T_{2}$ is less than that required, the difference will be compensated by the mechanical joint limit. For a fully open finger:

$T_{2}=R(0) T_{a}$,

where $R(0)$ is the transmission ratio for $\theta_{2}=0$, and:

$f_{2}=\frac{T_{a}}{l+k_{2}}$.

Hence the torque required at $\mathrm{O}_{2}$ is:

$T_{2}^{r}=\frac{T_{a} k_{2}}{l+k_{2}}$

and the mechanical joint limit torque $T_{m j l}$ is:

$T_{m j l}=\left(\frac{k_{2}}{l+k_{2}}+R(0)\right) T_{a}$.

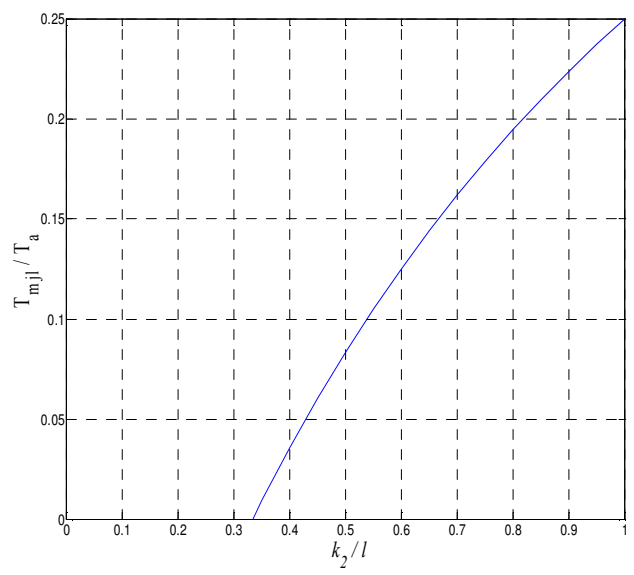

Fig. 7: Torque developed by the mechanical joint limit

The torque is linear according to $T_{a}$ and hyperbolic according to $k_{2}$. $T_{m j l}$ is a linear combination of $T_{a}$ and the torque required at $\mathrm{O}_{2}$ for the equilibrium. This latter is linear according to the contact force. Equation (1) shows that contact forces are linear with respect to $T_{a}$. Hence, $T_{m j l}$ is a linear combination of linear applications according to $T_{a}$. For a given acting torque, the required force for equilibrium is inversely proportional to the lever arm; hence the force is hyperbolic according to the lever arm. The contact force $f_{2}$ is the force that is required to equilibrating the finger when it is submitted to $T_{a} . T_{m j l}$ is linear according to $f_{2}$, hence it is hyperbolic according to lever arm $k_{2} . T_{m j l}$ remains zero until $k_{2}$ exceeds $l_{2} / 3$. Fig.7. shows that if a contact begins with $k_{2}$ less than $l_{2} / 3$ the sliding led to a stable grasp and never to a finger fully open. The maximum value of $T_{m j l}$ with respect to $T_{a}$ is the absolute value of $R(0) . T_{m j l}$ is maximum when the finger is blocked at its extremity. The moment developed at $O_{I}$ by a force $f$ applied on the extremity is the same that developed by two forces equal to $f$ applied at the middles of both phalanxes. $R(0) \cdot T_{a}$ matches that required to equilibrating a force applied at the middle of the phalanx. In order to equilibrate a force at the extremity, the half of the torque required at $\mathrm{O}_{2}$ is provided by the mechanical joint limits and the other half by the cam, which explains the result.

\section{Force in the tendon}

The load in the tendon depends on the actuator torque and on the contact point location on the phalanxes. In the case where the grasp is isotropic, we consider that both phalanxes are subjected to two equal forces $f$ on their middle. The force $T$ in the tendon is the ratio between the actuator torque and the radius of the pulley.

$\frac{T}{f}=\frac{T_{a}}{f r}=\left(1+\cos \theta_{2}\right) \frac{l}{r}$.

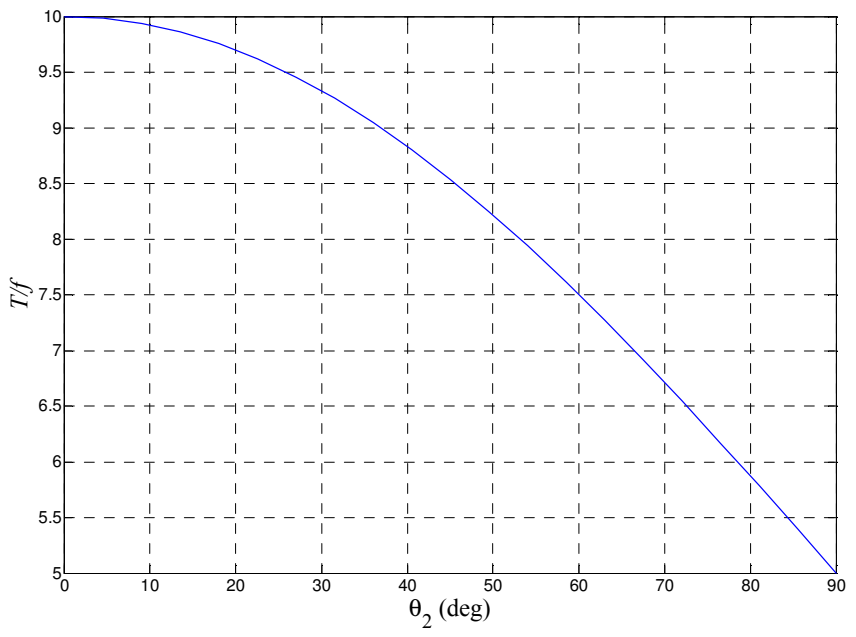

Fig. 8: Tension force in the tendon

The curve of Fig. 8 shows that the force in the tendon is between five and ten times the contact force at the isotropy. This result highlights the disadvantage of differential tendon mechanisms mentioned in Section 2. The result explains why the tendon mechanisms are used for soft grasp, and bar linkage mechanisms for heavy one. A high actuator torque stretches the tendon and may cut it. This feature can be used to limit contact forces and prevent the deterioration of the grasped object, breack it has also the disadvantage to loose the control of the gripper. 


\section{CONCLUSIONS AND FURTHER WORKS}

In this paper, we have realized a kinetostatic stability study of an isotropic underactuated two-phalanx finger. This analysis highlighted the grasp stability of this finger. We analysed the case of grasp with both phalanxes and the case of geasping with the only distal phalanx. From this study, we can conclude that ejection can never occur. After sliding, grasp is stable or the finger is fully open and blocked by the mechanical joint limits. Two main internal efforts in the finger were studied. These efforts are the torque developed by the mechanical joint limit and the force in the tendon. The torque developed by the mechanical joint limit depends as on the actuator torque as on the contact point location on the distal phalanx. The tension in the tendon is very high comparatively to the contact force, which explains why tendon mechanisms are prefered for soft grasp. In this paper, we presented also the design of the finger and the profile of the cam, which ensures the isotropy.

In the next future, we will validate our analysis on a prototype of the finger that is under construction. We also plane to complete this work by introducing more realistic physical parameters that have been ignored such as friction between the finger and the object as well as the tendon stretching.

\section{REFERENCES}

[1] S. Hirose and Y. Umetani, "The development of soft gripper for the versatile robot hand", Mechanism and Machine Theory, Vol. 13, pp. 351-358, 1978.

[2] L. Birglen and C. Gosselin, "Optimal Design of 2-Phalanx Underactuated Fingers", in Proc. of International Conference on Intelligent Manipulation and Grasp, pp. 110-116, Genoa, Italy, July 12,2004

[3] S. C. Jacobsen, E. K. Iversen, D. F. Knutti, R. T. Johnson, and K. B. Biggers, "Design of the UTAH/MIT dextrous hand", in Proc. of ICRA86, San Francisco, CA, USA, 1986, pp. 1520-1532.

[4] J. K. Salisbury and J. J. Craig, "Articulated hands: Force control and kinematic issues", The International Journal of Robotics Research, Vol. 1, No. 1, pp. 4-17, 1982.

[5] G. A. Bekey, R. Tomovic, and I. Zeljkovic, "Control Architecture for the Belgrade/USC Hand in Dextrous Robot Hands", Springer-Verlag, New York, 1999.

[6] J. Butterfass, M. Grebenstein, H. Liu, and G. Hirzinger, "DLR-hand II: Next generation of a dextrous robot hand", in Proc. of ICRA 2001, Seoul, Korea, May 21-26 2001, pp. 109-114.

[7] T. Laliberté, L. Birglen and C. Gosselin, "Underactuation in robotic grasp hands," Japanese Journal of Machine Intelligence and Robotic Control, Special Issue on Underactuated Robots, vol. 4, no. 3, pp. 7787, September 2002.

[8] H. Shimojima, K. Yamamoto and K. Kawawita, "A Study of Grippers with Multiple Degrees of Mobility," JSME International Journal, Vol. 30, No. 261, pp. 515-522, 1987.

[9] S.J. Bartholet, "Reconfigurable End Effector," U.S. Patent 5108 140, 1992.

[10] R.M. Crowder and D.R. Whatley, "Robotic Gripping Device Having Linkage Actuated Finger Sections," U.S. Patent 4834 443, 1989.

[11] A. Bicchi and V. Kumar, "Robotic grasp and contact: A review," in Proc. of ICRA2000, San Francisco, CA, USA, 2000.

[12] N. Fukaya, S. Toyama, T. Asfour and R. Dillmann, "Design of the TUAT/Karlsruhe Humanoid Hand", in IROS 2000, Takamatsu, Japan, October 30 - November 5, 2000.
[13] T. Laliberté and C. Gosselin, "Actuation System for Highly Underactuated Gripping Mechanism”, United States Patent, No. US 6,505,870 B1, January 14, 2003.

[14] B. Massa, S. Roccella, M. C. Carrozza and P. Dario, "Design and Development of an Underactuated Prosthetic Hand", in Proc. of ICRA2002, Washington, DC, May 2002.

[15] R. Olfati-Saber, "Nonlinear Control of Underactuated Mechanical Systems with Application to Robotics and Aerospace Vehicles", Ph.D. Thesis, Department of Electrical Engineering and Computer Science, Massachusetts Institute of Technology, Cambridge, MA, February 2001.

[16] W.T. Townsend, "The BarrettHand grasper - programmably flexible part handling and assembly", Industrial Robot: An International Journal, MCB University Press, Vol. 27, No. 3, pp. 181-188, 2000.

[17] W. Zhang, Q. Chen, Z. Sun and D. Zhao, "Under-actuated passive adaptive grasp humanoid robot hand with control of grasp force", in Proc. of ICRA2003, Taipei, Taiwan, September 14-19, 2003.

[18] N. T. Ulrich, "Methods and Apparatus for Mechanically Intelligent Grasp", United State Patent, No. US 4,957,320, 1988.

[19] S. Lee, "Artificial Dexterous Hand", United States Patent, No. US 4,946,380, August 7, 1990.

[20] S. Krut, "A Force-Isotropic Underactuated Finger", Proceeding of the 2005 IEEE, International Conference of the robotics and automation, Barcelona, Spain, April 2005

[21] Hirose S., "Connected Differential Mechanism and its Applications", in Proc. of ICAR85, Tokyo, Japan, September 1985.

[22] L. Birglen, "Analysis and control of underactuated robotic hand", Ph.D. thesis, university Laval, Quebec Canada.

[23] N. Eduardo, N, Rodriguez, G.Carbone, M. Ceccarelli, "Optimal design of driving in a 1-DOF anthropomorphic finger", Mechanism and Machine Theory, 2006, Vol 41, p897-911

[24] R. Olfati-Saber, "Fixed Point Controllers and Stabilization of the CartPole System and the Rotating Pendulum", proc. of the 38th Conference on Decision and Control, pp. 1174-1181, Phoenix, AZ, Dec. 1999.

[25] K.H. Hunt, Kinematic Geometry of Mechanisms, Clarendon Press, Oxford, 1978.

[26] J.D. Crisman, C. Kanojia and I. Zeid, "Graspar : A Flexible, Easily Controllable Robotic Hand," IEEE Robotics and Automation Magazine, pp. 32-38, June 1996.

[27] D.F. Graham, "Artificial Hand and Digit Therefor," U.S. Patent 5200 679, 1993.

[28] N. Dechev, W.L. Cleghorn and S. Naumann, "Multiple Finger, Passive Adaptive Grasp Prosthetic Hand," Mechanism and Machine Theory, Vol. 36, No. 10, pp. 1157-1173, 2001.

[29] J. Zhang, G. Guo and W.A. Gruver, "Optimal Design of a Six- Bar Linkage for an Anthropomorphic Three-Jointed Finger Mechanism," Proceedings of the ASME Mechanisms Conference, Phoenix, vol. DE45, pp. 299-304, 1992.

[30] E.N. Haulin, A.A. Lakis and R. Vinet, "Optimal Synthesis of a Planar Four-Link Mechanism Used in a Hand Prosthesis," Mechanism and Machine Theory, Vol. 36, Nos. 11-12, pp. 1203-1214, 2001.

[31] J. E. Shigley, "Mechanical engineering design" 5th edition, ISBN 007-100 607-9, 1989 